\title{
Ecuación para estimar la biomasa arbórea en los bosques tropicales de Costa Rica'
}

\author{
Equation for estimating tree biomass in \\ tropical forests of Costa Rica
}

\author{
Shu Wei Chou \\ Edgar E. Gutiérrez-Espeleta ${ }^{3}$
}

Fecha de recepción: 29 de julio del 2012 Fecha de aprobación: 16 de setiembre del 2012

Chou, S.W.; Gutiérrez-Espeleta, E. Ecuación

para estimar la biomasa arbórea en los bosques tropicales de Costa Rica. Tecnología en Marcha. Vol. 26, № 2. Pág 4I-54.

I Este estudio se enmarcó en el proyecto de investigación "Monitoreo de ecosistemas forestales para el fortalecimiento de estrategias de conservación y uso de bosques: una contribución a la iniciativa Costa Rica Carbono Neutral", coordinado por el Instituto de Investigación y Servicios Forestales (INISEFOR-UNA), con la participación del Centro de Investigación en Integración Bosque Industrial, del ITCR y la Escuela de Estadística de la UCR.

2 Bachiller en Estadística y estudiante graduado del Programa de Posgrado en Estadística. Taiwanés. vicchou325@yahoo.com.tw. Universidad de Costa Rica.

3 Catedrático de la Escuela de Estadística, Ph.D. en Biometría Forestal. Costarricense. Edgar.gutierrez@ucr.ac.cr. Universidad de Costa Rica.

Los autores le agradecen al Prof. Luis Acosta Vargas, del Departamento de Ingeniería Forestal del Instituto Tecnológico de Costa Rica (ITCR), por su colaboración en este artículo. Igualmente, al Prof. Fernando Ramírez, de la Escuela de Estadística de la Universidad de Costa Rica (UCR), por sus comentarios a una primera versión de este trabajo que se presentó en el curso de Práctica Profesional (XS-0422) de esta Escuela. 


\section{Palabras clave}

Estimación de almacenamiento de carbono; estimación de biomasa; bosques tropicales; densidad de la madera; Costa Rica.

\section{Resumen}

Una de las medidas más relevantes para la mitigación del cambio climático es la conservación y regeneración del bosque en nuestros países. La cantidad de carbono que se almacena en la biomasa arbórea pasa a ser una medida relevante para la política pública. El presente trabajo analiza la asociación que tienen algunas variables dasométricas, fácilmente medibles, asociadas a la biomasa, con el propósito de estimarla indirectamente, dado que la medición directa de la biomasa arbórea es un trabajo complejo y tiene un costo muy elevado.

El objetivo general del estudio fue hacer un análisis del comportamiento de las variables dasométricas fácilmente medibles para predecir biomasa arbórea con datos de dos bosques tropicales de Costa Rica, con el propósito de analizar su posible aplicación generalizada en los bosques tropicales de todo el país.

Mediante una revisión bibliográfica, se determinaron cuatro posibles modelos que estiman biomasa en bosques tropicales. Se evaluaron 907 árboles con diámetro a la altura de pecho (dap) mayor a $10 \mathrm{~cm}$ en dos bosques tropicales de Costa Rica (Parque Nacional Corcovado en el suroeste y Fila Carbón en el sureste, vertiente del Caribe), generando una estimación de biomasa lo más precisa posible. Se realizó un análisis de las variables de los árboles (densidad específica de la madera, altura total y dap) y su biomasa, con el fin de desarrollar el modelo que facilitara la predicción de esta. El modelo final utiliza como variables independientes el dap y la densidad. Con el dap se da el hecho de que existe una alta correlación con la altura total, la cual es muy difícil de obtener en el campo, de modo que se decidió no utilizarla. La variable densidad es importante, ya que dos árboles con la misma estructura pero distinta densidad van a mostrar diferente cantidad de biomasa. Para la estimación de este modelo se utilizó una regresión segmentada (por la relación que tienen el dap y la altura total con la variable biomasa transformada) y cuadrados medios ponderados para resolver el problema de heterocedasticidad. El modelo final cumplió con los supuestos estadísticos de una regresión lineal general, evaluados por el comportamiento de los residuos y otras pruebas paramétricas, y obtiene un coeficiente de determinación de 0,992.

Como conclusión, este estudio propone un enfoque metodológico para estimar la biomasa a nivel general en los bosques, lo cual se considera de utilidad para fundamentar la toma de decisiones sobre el almacenamiento a largo plazo del carbono en los bosques tropicales. Se espera que en estudios futuros se disponga de parcelas de otros bosques con mediciones de la biomasa real, para seguir calibrando el modelo propuesto para la estimación de la biomasa almacenada en los bosques tropicales.

\section{Key words}

Carbon storage estimation; biomass estimation; tropical forests; wood density; Costa Rica.

\begin{abstract}
One of the most relevant measures for climate change mitigation is the conservation and regeneration of forests in our countries. The amount of stored carbon in the forest biomass becomes a relevant variable for public policy. The present article analyses the association of some dasometric variables, easily obtained, with tree biomass with the purpose of indirectly estimating it, given that direct measurement of tree biomass is a complex and very expensive task.
\end{abstract}

The general objective of this study was to analyze the pattern shown by dasometric variables, easily obtained, with tree biomass to estimate it by using data from two tropical forests in Costa Rica, with the purpose of analyzing its potential general application in tropical forests in the entire country.

The bibliographic review helped to identify four possible models which estimate biomass in tropical forests. 907 trees were used with diameter at breast height (dbh) greater than $10 \mathrm{~cm}$ in two tropical forests in Costa Rica (Corcovado National Park at the SW of the country, and Fila Carbón at the SE part, Caribbean slope), generating a biomass estimate as precise as possible. Dasometric variables (wood density, total height and dbh) were used to 
analyze their relationship with biomass with the objective of finding a model that could predict it. The final model uses, as independent variables, $\mathrm{dbh}$ and density. Dbh has a high correlation with tree total height, which is very difficult to obtain in the field, therefore it was not used. The variable density is very important as it varies between trees having the same structure but of different species. To estimate the model, a segmented regression technique was used as well as weighted mean squares to solve for heteroscedasticity. The final model met all statistical assumptions for a linear model according to the residual and parametric analysis. The determination coefficient resulted in 0,992. As a conclusion, the study proposes a methodological approach to estimate forest biomass, which is very useful to support decision-making on carbon storage in tropical forests. Biomass determination in the field in future plots is going to help the calibration of this approach to estimate biomass stored in tropical forests.

\section{Introducción}

El cambio climático es un tema del que se habla con mucha frecuencia en la actualidad. Una gran cantidad de instituciones científicas, universidades, gobiernos y otras entidades se preocupan por este fenómeno debido a que el cambio que se está experimentando afecta no solo las dinámicas propias de los ecosistemas sino también las dinámicas de las poblaciones humanas. Se puede entender el cambio climático como "un fenómeno que se expresa como una desviación del tiempo meteorológico promedio esperado o de las condiciones climáticas normales (temperatura y precipitación) para un lugar y tiempo dados" (Dávalos et al., 2008, p. 224). La aceleración del cambio climático, preocupación fundamental en la actualidad, es causada por aquella actividad humana que, sin considerar la sostenibilidad de la especie, ha incrementado el uso de combustibles fósiles, los cambios en el uso de la tierra y en general aquellas actividades desarrolladas según principios de beneficio en el corto plazo. De acuerdo con el Grupo Intergubernamental de Expertos sobre Cambio Climático (IPCC) (2007), el aumento en la concentración en la atmósfera de gases como metano $\left(\mathrm{CH}_{4}\right)$, hidrofluorocarburos (HFCs), hexafluoruros de azufre ( $\mathrm{SF}_{6}$ ), perfluocarburos (PFCs), óxido nitroso $\left(\mathrm{N}_{2} \mathrm{O}\right)$ y principalmente dióxido de carbono $\left(\mathrm{CO}_{2}\right)$, todos ellos llamados "gases de efecto invernadero", se debe a las actividades humanas desde el año 1750.

Una de las alternativas de solución o mitigación de este problema proviene del conocimiento del ciclo del carbono. El carbono es secuestrado (almacenado) por la vegetación mediante los procesos de fotosíntesis. El carbono queda almacenado en aproximadamente un $50 \%$ en la biomasa, sobre todo en aquella de vegetación leñosa (arbórea). Este tipo de biomasa es un depósito importante de los derivados del carbono y contribuye al almacenamiento de carbono en el suelo por medio de la acumulación de la materia orgánica (Schneider 1989, citado por Dávalos et al., 2008). De ahí la importancia de los inventarios forestales y de las mediciones repetitivas en parcelas permanentes, lo cual permite hacer valoraciones que conduzcan a decisiones de política pública. Con el objeto de colaborar en esta tarea, se propuso como objetivo del presente estudio desarrollar una ecuación general para predecir biomasa arbórea arriba del suelo, con datos de dos bosques tropicales de Costa Rica a partir de variables del árbol fácilmente medibles.

\section{Principales aspectos metodológicos}

Como la biomasa es una variable difícil de estimar y aún hoy día no existe una ecuación que pueda aplicarse de forma generalizada para su estimación en el contexto tropical, inicialmente se realizó una revisión bibliográfica para determinar y seleccionar aquellas ecuaciones que, por su naturaleza y estructura, podrían utilizarse para el ejercicio propuesto. Se encontraron cuatro modelos, que se presentan más adelante, los cuales se evaluaron con datos para diámetro a 1,3 m de altura y altura total de árboles en dos bosques tropicales ${ }^{4}$ y datos sobre la densidad de la madera de las distintas especies encontradas en ellos. ${ }^{5}$ Al final se construyó un modelo estadístico generalizado para estimar la biomasa.

Los dos bosques tropicales se encuentran en:

- Península de Osa, Parque Nacional Corcovado, Estación Sirena: se caracteriza por ser un bosque

4 Datos proporcionados por el Dr. Ruperto Quesada Monge, del Centro de Investigación en Integración Bosque Industrial (CIIBI) del ITCR.

5 Datos proporcionados por la Licda. Isabel Carpio Malavassi, del Laboratorio de Productos Forestales de la Facultad de Ingeniería de la UCR. 
muy húmedo tropical, con precipitaciones mayores a los $6000 \mathrm{~mm}$ anuales y una temperatura media de unos $26^{\circ} \mathrm{C}$. Se seleccionaron los árboles que tienen un dap $>10 \mathrm{~cm}$. Las parcelas están entre los 5 y 40 metros sobre el nivel del mar (msnm). Una de las parcelas utilizadas se encuentra en un bosque primario (Sirena BP) y la otra en un bosque secundario (Sirena BS).

- Caribe Sur,Talamanca, Fila Carbón: se caracteriza por ser un bosque húmedo tropical con precipitaciones de unos $5000 \mathrm{~mm}$ anuales y una temperatura media de $24^{\circ} \mathrm{C}$. Las parcelas están entre los 30 y 200 msnm. Las parcelas utilizadas en este bosque se denominan Fila Carbón y corresponden a un bosque primario y secundario intervenido.

Las parcelas en ambos sitios tienen la siguiente información dasométrica para árboles: diámetro a la altura del pecho, altura total expresada en metros, altura comercial expresada en metros. Para la densidad de los árboles por especie, se consultó al Laboratorio de Productos Forestales de la Universidad de Costa Rica.

De acuerdo con Brown (1997), existen dos métodos para estimar la biomasa de un bosque. El primer método es destructivo y consiste en cortar los árboles y pesar la biomasa directamente secando la madera. El segundo método es estimar la biomasa de manera indirecta por medio de modelos de regresión con variables obtenidas en el campo. Es evidente que el primer método, aunque muy preciso, no es factible para el presente estudio ya que para medir la biomasa se destruyen los árboles, por lo que este estudio se concentra en la construcción y comparación de modelos de regresión para la estimación de biomasa.

Al carecer de datos sobre biomasa, y de posibilidades reales de obtenerlos, se decidió estudiar los modelos más utilizados en la literatura forestal tropical para predecir esta variable, con el objeto de determinar un mejor criterio predictivo convergente de esta variable para cada árbol en los dos bosques lluviosos de Costa Rica bajo estudio (Fase I). El cuadro I presenta los cuatro modelos seleccionados que estiman la biomasa total arriba del suelo. En la sección de resultados se mostrarán las comparaciones entre estos modelos.

Posteriormente, se procedió a ajustar un modelo de regresión lineal general con el fin de predecir la biomasa utilizando la menor cantidad de variables posibles (Fase 2). En todo el proceso de análisis se utilizó el programa estadístico $\mathrm{R}$.

Con el propósito de encontrar una cifra de biomasa total por hectárea en los bosques estudiados, se procedió a utilizar esta ecuación para predecir la biomasa de aquellos árboles con densidad conocida. Una vez obtenida, se procedió a construir su distribución diamétrica y para cada clase se obtuvo un promedio de biomasa. Como aún existían árboles con densidad desconocida, y por consiguiente esta ecuación no podía aplicarse en ellos, se procedió a estimar su biomasa aplicando el promedio de la clase diamétrica correspondiente a cada uno de ellos (Fase 3).

Cuadro I. Modelos predictivos de biomasa (kg por árbol) para bosques húmedos

\begin{tabular}{|l|l|}
\hline Modelo & \\
\hline$I^{\prime}$ & $\mathrm{B}_{1}=(\mathrm{d}) \cdot \mathrm{e}^{\left(-1,239+1,980 \cdot \operatorname{Ln}(\mathrm{dap})+0,207 \cdot(\ln (\mathrm{dap}))^{2}-0,0281 \cdot(\ln (\mathrm{dap}))^{3}\right)}$ \\
\hline $2^{\prime}$ & $\mathrm{B}_{2}=\mathrm{e}^{(-2,557+0,94 \cdot \ln (\mathrm{d} \cdot \mathrm{dap} \cdot \mathrm{ht}))}$ \\
\hline $3^{2}$ & $\mathrm{~B}_{3}=21,297-6,953 \cdot(\mathrm{dap})+0,740 \cdot(\mathrm{dap})^{2}$ \\
\hline $4^{3}$ & $\left.\mathrm{~B}_{4}=0,06 \cdot \mathrm{d} \cdot\left((\pi \cdot \mathrm{dap})^{2}\right) / 4\right) \cdot h t$ \\
\hline
\end{tabular}

Nota: $\mathrm{B}=$ biomasa aérea $(\mathrm{kg})$, dap = diámetro a la altura del pecho $(\mathrm{cm}), \mathrm{d}=$ peso específico básico $\left(\mathrm{g} / \mathrm{cm}^{3}\right)$, ht $=$ altura total (metros)

Fuente: 'Tree allometry and improved estimation of carbon stocks and balance in tropical forests (Chave et al., 2005). ${ }^{2}$ IPCC 2003, citando a Brown 1997; Brown y Schroeder 1999; Schroeder et al., 1997. 'Forma estándar de estimación de biomasa por medio del volumen de un árbol según Chave et al., 2005. 


\section{Resultados}

En el cuadro 2 se resume la información básica de las parcelas en ambos sitios estudiados. Además, es importante mencionar que para Sirena BP y Sirena BS se recolectaron datos de árboles que tienen dap $>5 \mathrm{~cm}$, pero para Fila Carbón se recolectaron datos de árboles que tienen dap $>10 \mathrm{~cm}$, dadas las necesidades y recomendaciones del proyecto de Monitoreo de Ecosistemas Forestales. Para este estudio se decidió excluir los árboles que tienen d de $5 \mathrm{~cm}$ a $10 \mathrm{~cm}$ para estandarizar los árboles de los dos bosques respecto a esta medición.

El 68,5\% de los árboles encontrados en Sirena BS tienen datos para densidad mientras que Sirena BP tiene solo un 19,8\% de los árboles con densidad conocida, de forma tal que un $42,6 \%$ de los árboles de las parcelas estudiadas se incluyeron en el análisis, o sea, 907 árboles de un total de 2.129.

Figura I. Datos de densidad en árboles

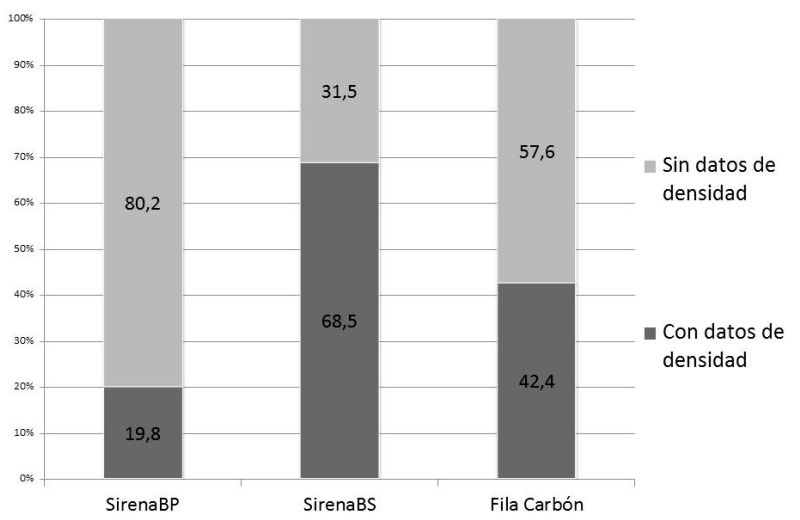

\section{Resultado de la Fase I: Comparación de los cuatro modelos en la estimación de biomasa}

De los modelos seleccionados de la literatura, es necesario determinar cuáles estiman mejor la biomasa utilizando variables dasométricas fácilmente obtenibles en el campo. Para este fin, se utilizaron las mediciones de los árboles de dos bosques de la zona sur del país, tal como se observa en el cuadro I. Un resumen de los modelos seleccionados se presenta en el cuadro 2, apreciándose que el modelo 2 y 4 ocupan las tres variables, para el modelo I se necesita la variable dap y densidad, mientras que el modelo 3 solo se estima por medio del dap. Este modelo 3 es el modelo más sencillo y más práctico pues solo se necesita del dap.

El cuadro 3 resume las correlaciones de los resultados de la aplicación de los cuatro modelos. Se observan claramente las altas correlaciones entre los resultados.

Cuadro 3 Variables que utilizan los modelos para estimar la biomasa

\begin{tabular}{|c|c|c|c|}
\hline Modelo & dap & $d$ & ht \\
\hline I & $\sqrt{ }$ & $\sqrt{ }$ & \\
\hline 2 & $\sqrt{ }$ & $\sqrt{ }$ & $\sqrt{ }$ \\
\hline 3 & $\sqrt{ }$ & & \\
\hline 4 & $\sqrt{ }$ & $\sqrt{ }$ & $\sqrt{ }$ \\
\hline
\end{tabular}

Fuente: Elaboración propia.

Cuadro 2. Características básicas de las parcelas estudiadas según tipo de bosque

\begin{tabular}{|c|c|c|c|c|c|c|}
\hline \multirow[t]{2}{*}{ Lugar } & \multirow[t]{2}{*}{ Tipo de bosque } & \multirow{2}{*}{$\begin{array}{l}\text { Número de } \\
\text { parcelas }\end{array}$} & \multirow{2}{*}{$\begin{array}{l}\text { Año de } \\
\text { recolección }\end{array}$} & \multirow{2}{*}{$\begin{array}{l}\text { Total de } \\
\text { árboles }\end{array}$} & \multicolumn{2}{|c|}{$\begin{array}{c}\text { Total de árboles con densidad } \\
\text { específica conocida' }\end{array}$} \\
\hline & & & & & Total & $\%$ del total \\
\hline Sirena BP & Bosque primario & I & 2006 & 445 & 88 & 19,8 \\
\hline Sirena BS & Bosque secundario & I & 2006 & 403 & 276 & 68,5 \\
\hline Fila Carbón & $\begin{array}{l}\text { Bosques secundarios y primarios } \\
\text { intervenidos }\end{array}$ & 8 & 2007 & $128 \mid$ & 543 & 42,4 \\
\hline & & Total & & 2129 & 907 & 42,6 \\
\hline
\end{tabular}

' Las especies con información sobre la densidad de su madera son las que se incluyen en el estudio.

Fuente: Elaboración propia. 
Cuadro 4. Matriz de correlaciones de Pearson entre las estimaciones de biomasa de los cuatro modelos

\begin{tabular}{|c|c|c|c|}
\hline Modelo & 2 & 3 & 4 \\
\hline 1 & 0,9906 & 0,9613 & 0,9876 \\
\hline 2 & & 0,9547 & 0,9981 \\
\hline 3 & & & 0,9623 \\
\hline 4 & & & \\
\hline
\end{tabular}

Fuente: Elaboración propia.

El mismo comportamiento se puede observar en la figura 2, en la cual se presentan varios gráficos de dispersión de todas las combinaciones posibles de los cuatros modelos. Los modelos 2 y 4 muestran una correlación casi perfecta (ambos modelos utilizan dap, d y ht), mientras que para el gráfico de dispersión del modelo 3 (utiliza solo dap) con los otros modelos se aprecia estimaciones aceptables para cantidades pequeñas de biomasa, pero no así para aquellas de biomasa mayores. Este análisis lleva a la misma conclusión: que el modelo 3 se aleja más de los otros tres modelos.
Despejando las ecuaciones de los modelos 2 y 4 se obtienen las siguientes ecuaciones o nuevos modelos:

$$
\begin{aligned}
& B_{2}=0,07754 \cdot\left(d \cdot d a p^{2} \cdot h t\right)^{0,94} \\
& B_{4}=0,047 \mid 2 \cdot\left(d \cdot d a p^{2} \cdot h t\right)^{\prime}
\end{aligned}
$$

Como se puede observar en las dos ecuaciones anteriores, la diferencia en los exponentes es casi mínima (0,94 vs I), por lo que estos dos modelos difieren básicamente por el valor de la constante, siendo $B_{2} 1,65$ veces mayor que $B_{4}$. Esto causa que la estimación del modelo 2 sea menos dispersa (estimaciones "conservadoras" versus estimaciones "optimistas") que la del modelo 4, es decir, con un árbol grande con la estimación de modelo 4 se estimaría más biomasa que con el modelo 2. Y si es un árbol pequeño, con la estimación del modelo 4 se va a estimar menos biomasa que con el modelo 2 .

La ecuación 3, B3 = $21,297-6,953 \cdot($ dap $)+0,740 \cdot(\text { dap })^{2}$ es una ecuación cuadrática cóncava hacia arriba. Es obvio que al aumentar la medida del dap de un árbol, aumentará la estimación de la biomasa. Es por eso que la biomasa estimada del modelo 3 presenta una sobreestimación para los árboles con dap grande.

Figura 2. Gráficos de dispersión de los resultados de los cuatro modelos
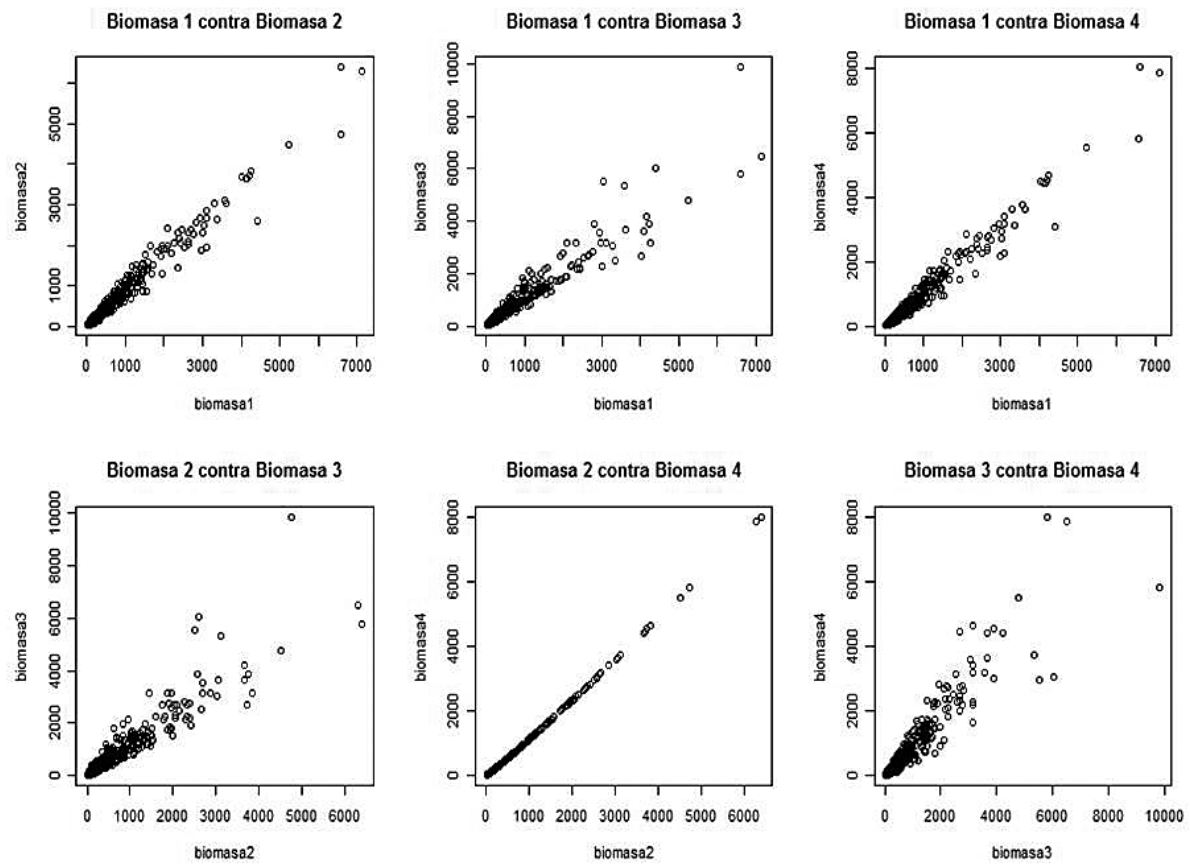

Fuente: Elaboración propia. 
Por lo tanto, se decidió utilizar el promedio de los modelos 2 y 4 para estimar la biomasa de los árboles en las parcelas seleccionadas. Se entiende que la única forma de corroborar el error de estimación sería contrastando estas estimaciones con los datos reales observados de biomasa. Sin embargo, para efectos de estudio, bastó que las estimaciones de los modelos seleccionados fueran estadísticamente similares para continuar con la investigación. Teniendo una estimación de biomasa para cada árbol, se procedió a ajustar un modelo de regresión que mejor ajustara la variable biomasa a partir de variables dasométricas fáciles de obtener y de bajo costo para la estimación. Por ejemplo, la altura total es una variable difícil de obtener debido al efecto de copa y la dificultad de medirla por la abundancia de individuos que caracterizan a los bosques tropicales.

\section{Fase II: ajuste del modelo de regresión lineal general}

\section{Análisis exploratorio}

El cuadro 5 presenta los coeficientes de correlación de Pearson de las variables observadas en las parcelas seleccionadas. La figura 3 muestra los gráficos de dispersión de las relaciones entre variables. De esto se desprende que las variables más correlacionadas con la biomasa son la variable dap y la altura total; además, la densidad específica de la madera tiene una baja correlación con la biomasa.

La relación de dap con la biomasa no es lineal, tal como se observa en la figura 3 (una curva se ajusta mejor que una línea). La densidad, al igual que el resultado de las correlaciones de Pearson, no presenta ningún patrón en el gráfico. Con fundamento en estos resultados del análisis exploratorio, se decidió utilizar las variables dap y altura total para ajustar el modelo. Las razones para esta decisión son las siguientes:

- Debido a que la altura total es compleja de medir en bosques tropicales, se ha encontrado que existe una relación directa significativa entre dap y la altura total del árbol. Esta relación muestra que los árboles en bosques húmedos o pluviales crecen en dap y altura total rápidamente, dentro del rango de diámetro de 30-50 cm aproximadamente. Después de 50 $\mathrm{cm}$ de dap, la altura total empieza a crecer cada vez más lenta, tal como se observa en el gráfico de dispersión de altura total y dap en la figura 3.

- Aunque la correlación de la densidad con la biomasa no es significativa, la densidad sigue jugando un papel importante que influye en la cantidad de biomasa. Por ejemplo, en el caso de dos árboles con igual volumen (mismo dap y misma altura) pero con densidades diferentes, sus cantidades de biomasa serán distintas.

- Tras el análisis exploratorio se decidió utilizar una regresión segmentada para estimar biomasa utilizando la variable dap con los puntos de cambio en 30 y $50 \mathrm{~cm}$, pues se espera tener diferentes comportamientos en la producción de biomasa entre árboles en las distintas clases diamétricas y la variable densidad.

\section{Ajuste del modelo}

El análisis exploratorio demostró que no existe una relación lineal significativa entre las variables dap y densidad, como variables explicativas, y la biomasa, como variable estimada. Si se ajustara el modelo lineal, no cumpliría los supuestos de una regresión lineal múltiple. Por lo tanto, se decidió utilizar el método de cuadrados medios ponderados utilizando la

Cuadro 5 Matriz de correlaciones entre la variable biomasa y las variables dasométricas dap, densidad y altura total

\begin{tabular}{|c|c|c|c|}
\hline Variables & dap & Densidad & Altura total \\
\hline Biomasa & 0,9129 & 0,0804 & 0,6493 \\
\hline dap & & $-0,0385$ & 0,7558 \\
\hline Densidad & & & 0,0307 \\
\hline
\end{tabular}

Fuente: Elaboración propia. 
Figura 3. Gráficos de dispersión entre la variable biomasa y las variables dap, densidad y altura total
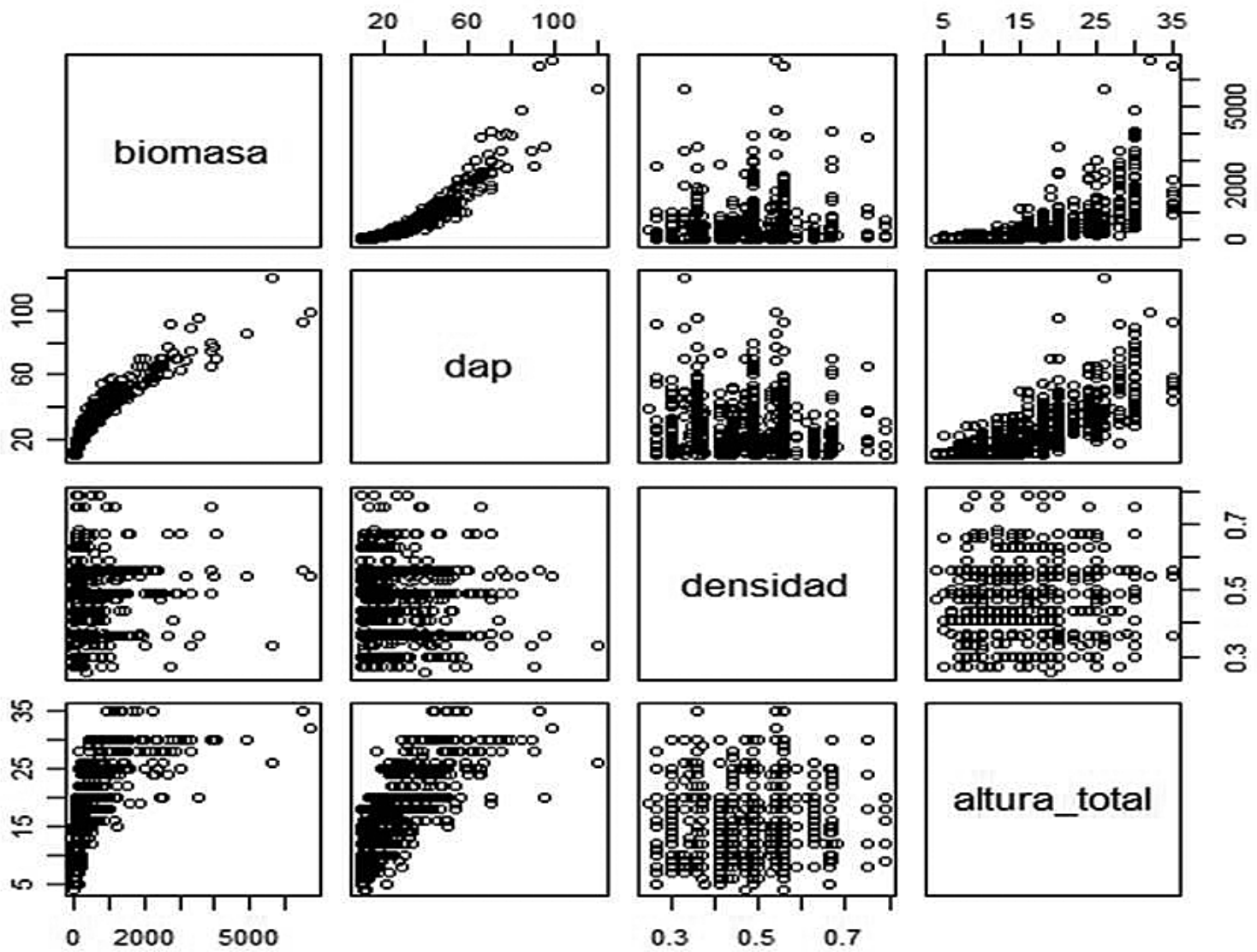

$\begin{array}{lll}0.3 & 0.5 & 0.7\end{array}$

Fuente: Elaboración propia.

variable biomasa transformada para lograr un buen ajuste. Se utilizó el método de transformación de Box-Cox para decidir la transformación requerida en la variable biomasa para ajustar el modelo.

En la figura 4 se aprecia que es apropiado realizar una transformación matemática de "raíz" de la variable biomasa. La prueba de los modelos se inició realizando transformaciones sencillas como raíz cuadrada, raíz cúbica, etc.; sin embargo, con ninguna de las transformaciones usuales se cumplían los supuestos de homoscedasticidad y normalidad de residuos de un modelo de regresión. Una transformación adecuada, observando el gráfico, fue 25.

El modelo de regresión segmentada que resultó apropiado fue el siguiente:

$B^{2 / 5}=\beta_{0}+\beta_{1} \cdot \operatorname{dap}+\beta_{2} \cdot \delta+\beta_{3} \cdot \operatorname{dap} \cdot \delta+\beta_{4}\left(\operatorname{dap}-\varphi_{1}\right)$ $X_{1}+\beta_{5}\left(\right.$ dap $\left.-\varphi_{2}\right) X_{2}$ (Ec. 5)
Donde:

$\mathrm{B}=$ biomasa $(\mathrm{kg})$

dap = diámetro $(\mathrm{cm})$ medido a 1,3 $\mathrm{m}$ de altura

$\delta=$ peso específico básico $(\mathrm{g} / \mathrm{cm} 3)$

$\varphi_{1}=30$ (primer punto de cambio)

$\varphi_{2}=50$ (segundo punto de cambio)

$X_{1}=$ variable dicotómica ( 1 si dap $\geq 30 \mathrm{~cm}$ y 0 si dap $<30 \mathrm{~cm}$ )

$X_{2}=$ variable dicotómica (I si dap $\geq 50 \mathrm{~cm}$ y 0 si dap $<50 \mathrm{~cm}$ )

\section{Verificación de supuestos}

Los supuestos que tiene un modelo de regresión son: homoscedasticidad (variabilidad constante de los errores), normalidad de los errores, indepen- 
Figura 4. Transformación de Box-Cox

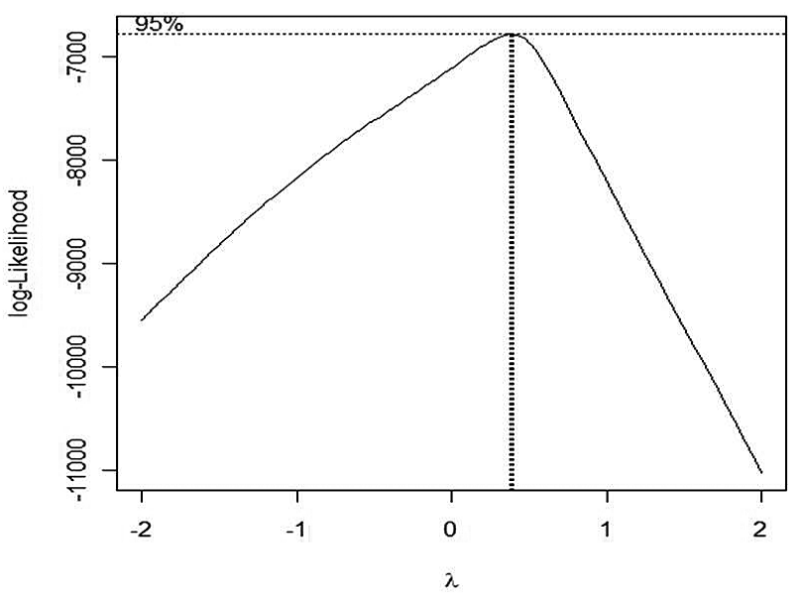

dencia lineal, linealidad. El supuesto de homoscedasticidad se logró en el ajuste, obteniendo un valor de significancia de 0,1 I con la prueba de variancia constante (la prueba formal de Breusch-Pagan), por lo que se concluyó, con un nivel de significancia de 0,05 , que no hay suficiente evidencia para rechazar la hipótesis nula de variancia constante.

Para el supuesto de normalidad se utilizó los QQ-plots. Aunque presenta unos puntos desviados en los valores bajos, una muestra grande hace que los coeficientes del modelo se aproximen a la normalidad (los gráficos de los residuos y los QQ-plots se adjuntan en el anexo).

Para el supuesto de independencia lineal en el análisis exploratorio, se encontró que el coeficiente de correlación de Pearson no es significativo entre las dos variables explicativas. Mientras que para el supuesto de linealidad, con la transformación de la variable dependiente, se logró linealizar la relación (ver el anexo).

Adicionalmente, se determinaron varios valores extremos y casos de influencia, los cuales son árboles con dap grande $(>100 \mathrm{~cm})$ pero con densidades menores de $0,3 \mathrm{~g} / \mathrm{cm}^{3}$. Como estos árboles, con volúmenes muy grandes, juegan un papel importante en la conservación de la biomasa en los bosques, se decidió mantenerlos en el análisis.

El Cuadro 6 presenta el análisis de variancia del modelo. De dichas cifras se concluye que el modelo es apropiado con excepción de (el primer punto de cambio, dap $=30 \mathrm{~cm}$ ). Por lo tanto, se decidió excluirlo del modelo.

\section{Modelo final}

El modelo final seleccionado es el siguiente, eliminando el punto de cambio dap $=30 \mathrm{~cm}$ :

$$
B^{2 / 5}=\beta_{0}+\beta_{1} \cdot \delta+\beta_{2} \cdot \text { dap } \cdot \delta+\beta_{3}(\text { dap }-50) X
$$

Donde:

$$
\begin{aligned}
& \text { B = biomasa }(\mathrm{kg}) \\
& \text { dap = diámetro }(\mathrm{cm}) \text { medido a I,3 } \mathrm{m} \text { de altura } \\
& \delta=\text { peso específico básico }\left(\mathrm{g} / \mathrm{cm}^{3}\right) \\
& X=\text { variable dicotómica }(\mathrm{I} \text { si dap } \geq 50 \mathrm{~cm} \text { y } 0 \text { si } \\
& \text { dap }<50 \mathrm{~cm})
\end{aligned}
$$

\begin{tabular}{|c|c|c|c|c|c|}
\hline Fuente de error & g.l. & $\begin{array}{l}\text { Sum de } \\
\text { cuadrados }\end{array}$ & $\begin{array}{c}\text { Cuadrados } \\
\text { medios }\end{array}$ & Valor F & $\operatorname{Prob}(>\mathrm{F})$ \\
\hline$\Delta$ & I & 349,6 & 349,6 & 2451,6 & $<2,2 \mathrm{e}-16$ \\
\hline Dap & 1 & 23731,3 & 23731,3 & 166410,0 & $<2,2 \mathrm{e}-16$ \\
\hline$\left(\operatorname{dap}-\varphi_{1}\right) X_{1}$ & I & 74,2 & 74,2 & 520,3 & $<2,2 \mathrm{e}-16$ \\
\hline$\left(\operatorname{dap}-\varphi_{2}\right) X_{2}$ & 1 & 43,7 & 43,7 & 306,6 & $<2,2 \mathrm{e}-16$ \\
\hline$\varphi_{1}$ & I & 0,1 & 0,1 & 1,0 & 0,3291 \\
\hline$\varphi_{2}$ & 1 & 7,0 & 7,0 & 49,2 & $<4,57 \mathrm{E}-12$ \\
\hline$\Delta^{*}$ dap & I & 218,7 & 218,7 & 1533,8 & $<2,2 \mathrm{e}-16$ \\
\hline Residuals & 897 & 127.9 & 0.1 & & \\
\hline
\end{tabular}

Cuadro 6. Análisis de variancia del modelo con dos puntos de cambio 
La evaluación estadística de los supuestos resultó similar a la del modelo anterior, pues lo que se eliminó fue un punto de cambio que no era significativo y por lo tanto no afecta el modelo (ver los gráficos en el anexo).

En el cuadro 7 se resume el análisis de variancia del modelo final, apreciándose que todas las variables explicativas utilizadas aportan al modelo (a un nivel de significancia de la prueba de 5\%).

El cuadro 8 presenta las estadísticas de los coeficientes estimados del modelo. Se puede ver que los cuatro coeficientes resultaron significativos, con un nivel de significancia menor a 0,05. Por lo tanto, se biomasa obtenida en la Fase I. Este gráfico muestra que al aumentar el dap de un árbol, la variable densidad (utilizada en el modelo de estimación) empieza a jugar un papel más importante para estimar biomasa. Mientras que para árboles con dap $<50$ $\mathrm{cm}$ las estimaciones que utilizan solo dap y aquellas que usan dap y densidad son muy parecidas; para árboles con dap $>50 \mathrm{~cm}$ la situación es distinta. En la figura 5 se puede observar cómo, al aumentar el dap, las estimaciones de biomasa basadas solo en la medición del diámetro empiezan a alejarse de aquellas que además del dap utilizan la densidad específica de la madera, estando estas últimas más

Cuadro 7. Análisis de variancia del modelo con un punto de cambio

\begin{tabular}{|l|r|r|r|r|r|}
\multicolumn{1}{|c|}{ Fuente de error } & g.l. & $\begin{array}{c}\text { Suma de } \\
\text { cuadrados }\end{array}$ & Cuadrados medios & valor $F$ & \multicolumn{1}{c|}{$\operatorname{Pr}(>F)$} \\
\hline dap & 1 & 155944 & 155944 & 105654,2 & $<2,20 \mathrm{E}-16$ \\
\hline$\delta^{*}$ dap & 1 & 6765 & 6765 & 4583,5 & $<2,20 \mathrm{E}-16$ \\
\hline$\left(\right.$ dap $\left.-\varphi_{2}\right) X_{2}$ & 1 & 283 & 283 & 191,9 & $<2,20 \mathrm{E}-16$ \\
\hline Residuals & 901 & 1330 & 1 & & \\
\hline
\end{tabular}

Cuadro 8. Resumen de las estadísticas de los coeficientes del modelo final

\begin{tabular}{|c|c|c|c|c|}
\hline Coeficientes & Estimate & Error estándar & Valor $\mathrm{t}$ & $\operatorname{Pr}(>|t|)$ \\
\hline Intersección & 0,1438 & 0,02566 & 5,60 & 2.77E-08 \\
\hline dap & 0,2051 & 0,00256 & 80,12 & $<2 \mathrm{e}-16$ \\
\hline$\delta$ *dap & 0,3216 & 0,00482 & 66,76 & $<2 \mathrm{e}-16$ \\
\hline$\left(\operatorname{dap}-\varphi_{2}\right) \times_{2}$ & $-0,0744$ & 0,00537 & $-13,86$ & $<2 \mathrm{e}-16$ \\
\hline
\end{tabular}

decidió seleccionar el modelo con un solo punto de cambio como el mejor para este análisis. Además de cumplir los supuestos necesarios para un modelo de regresión lineal, el coeficiente de determinación, $R^{2}$, es 0,992 .

El comportamiento del gráfico entre la biomasa estimada de cada árbol utilizando el modelo anterior contra la biomasa obtenida en la Fase I, muestra una alta correlación lineal como era de esperar (ver figura 5). En la figura 6 se muestran las relaciones entre el dap, la biomasa estimada del modelo y la cercanas a las estimaciones de biomasa cuando se utiliza el modelo de la Fase II asumiendo una densidad igual a 0,5, según lo sugiere el IPCC para cuando se desconoce la densidad específica de la madera. $^{6}$

6 El IPCC ha sido enfático sobre la necesidad de que los países desarrollen investigaciones tendientes a encontrar los valores de densidad específica de la madera de sus propias especies arbóreas. 
Figura 5. Dispersión de la biomasa estimada vs. la biomasa obtenida

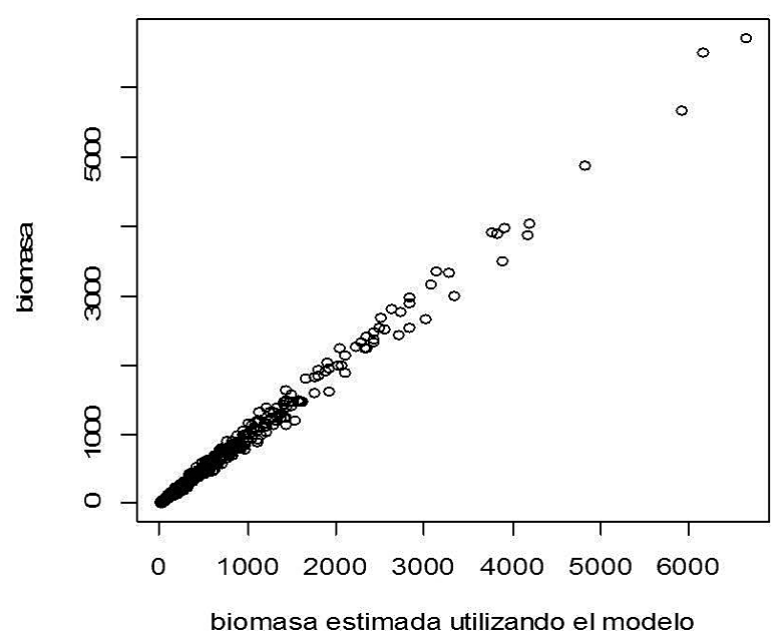

El modelo ajustado para predecir la biomasa viene dado por:

$B^{2 / 5}=0,|438+0,205| \cdot d a p+0,3216 \cdot d a p \cdot \delta$

$-0,0744($ dap -50$) X$

Donde:

$$
\begin{aligned}
& \mathrm{B}=\text { biomasa }(\mathrm{kg}) \\
& \text { dap }=\text { diámetro }(\mathrm{cm}) \text { medido a } \mathrm{I}, 3 \mathrm{~m} \text { de altura } \\
& \delta=\text { peso específico básico }\left(\mathrm{g} / \mathrm{cm}^{3}\right) \\
& X=\text { variable dicotómica }(\mathrm{I} \text { si dap } \geq 50 \mathrm{~cm} \text { y } 0 \text { si } \\
& \text { dap }<50 \mathrm{~cm})
\end{aligned}
$$

Así, se estima que para el bosque primario en Sirena se tiene una biomasa de 24l,15 ton ha ${ }^{-1}$, para el bosque secundario de este mismo sitio es de 142,37 ton ha-1 y para el bosque en Talamanca (intervenido) de 181,59 ton ha $^{-1}$. Sí se debe mencionar que para el primero solo se tenía información de densidad para el 19,8\% de los árboles, para el segundo el $68,5 \%$ y para el tercero el $42,4 \%$, según se indicó en Cuadro 2. Si se hubiera utilizado la recomendación del IPCC de 0,5 en densidad para aquellas especies con densidad desconocida, la biomasa total por hectárea de estos bosques hubiera sido de 266,06 ton ha-1, | 49,32 ton ha-1 y 184,32 ton ha-1, respectivamente. Sin embargo, observando la variabilidad de la densidad en estos bosques, la figura de densidad $=0,5$ pareciera no ser muy recomendable si se tienen porcentajes muy altos de especies con
Figura 6. Estimación de la biomasa arbórea a partir de mediciones del dap

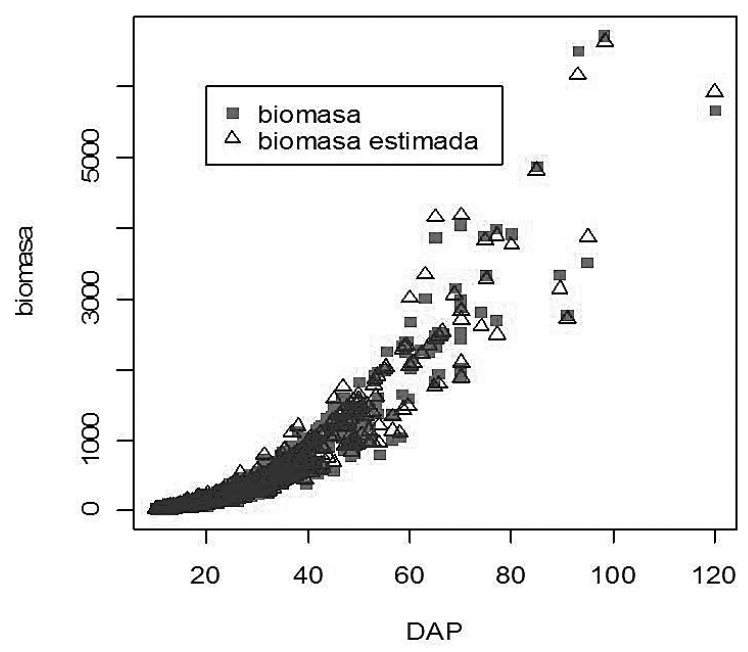

densidad desconocida, ya que, si bien el promedio ponderado de las densidades de las especies conocidas se encuentra alrededor de 0,5, los coeficientes de variación son relativamente altos (>25\%), lo cual significa que esta cifra no es una buena medida de sustitución.

\section{Conclusiones}

Los estudios relacionados con estimación de biomasa en Costa Rica, en su mayoría, solo se han realizado para unas pocas especies. Esto es una limitación para efectos de estimar la biomasa almacenada en los bosques tropicales, de ahí la necesidad de desarrollar un modelo que estime la biomasa en general, es decir, que no dependa de la especie. Si bien el procedimiento seleccionado para predecir inicialmente la biomasa introduce un error no medible, se consideró que esta era la mejor forma de proceder para obtener el dato de biomasa para una masa forestal tropical.

Este estudio representa un primer paso para identificar modelos en la estimación de biomasa en los bosques de Costa Rica. En el desarrollo del modelo presentado en este artículo encontramos algunas limitaciones, tales como la disponibilidad de datos observados sobre biomasa y la reducida cantidad de parcelas con datos disponibles. Además, la calidad de las pocas bases de datos disponibles nos 
llevó a detectar inconsistencias en ellas, sobre todo en las variables altura total y el dap. Otra limitación de importancia fue la escasa información sobre densidad de la madera para las especies de árboles en Costa Rica, a pesar del esfuerzo realizado por el Laboratorio de Maderas de la UCR, entre otras iniciativas.

Para comprobar la veracidad de la norma sugerida por el IPCC, se tomaron solo los árboles con densidad de la madera conocida de las tres parcelas bajo estudio. Se utilizó la Ec. 7 para calcular la biomasa y luego se usó el criterio del IPCC en los mismos árboles. El resultado obtenido indica que para la parcela del bosque primario en Sirena y para la ubicada en Fila Carbón, Talamanca, existen diferencias significativas (>10\%) para las estimaciones de biomasa entre estos dos métodos, pero no sucede lo mismo con la parcela de bosque secundario en Sirena, Osa. Este resultado es una invitación a seguir estudiando este tema en otros tipos de bosques tropicales.

Los resultados obtenidos en este estudio sirven para determinar una estimación de la cantidad de biomasa sobre el suelo en los bosques a partir de variables dasométricas comúnmente tomadas en el campo y del conocimiento sobre las propiedades de la madera, lo cual permitirá entender con más claridad la capacidad de estos para almacenar el carbono a largo plazo. Además, permitirá utilizar dicha información en la planificación de los inventarios forestales del país.

\section{Bibliografía}

Brown S. (1997). Estimating biomass and biomass change of tropical forest. FAO Forestry paper 134. Italia.

Carpio, I., Blanco, M., Cruz, L., Altamirano, J. \& Rivera, D. (1985). Estudio de 10 especies forestales de Sarapiquí, Informe final de proyecto. Laboratorio de Productos Forestales, Universidad de Costa Rica.

Carpio, I. (2003). Maderas de Costa Rica: 150 especies forestales. San José: Editorial de la Universidad de Costa Rica.

Chave, J., Andalo, C., Brown, S., Cairns, M., Chambers, J., Eamus, D., Fölster, H., Fromard, F., Higuchi, N., Kira, T., Lescure, J., Nelson, B., Ogawa, H., Puig, H., Riéra, B. \& Yamakura, T. (2005). Tree allometry and improved estimation of carbon stocks and balance in tropical forests. Oecologia Vol. I45, pp. 87-99.

Cubero, J. \& Rojas, S. (1999). Fijación de carbono en plantaciones de melina (Gmelina arborea Roxb.), teca (Tectona grandis L.f:) y pochote (Bombacopsis quinata Jaca.) en los cantones de Hojancha y Nicoya, Guanacaste, Costa Rica. Tesis de Licenciatura en Ciencias Forestales con Énfasis en Desarrollo Forestal. Facultad de Ciencias de la Tierra y el Mar. Escuela de Ciencias Ambientales. Universidad Nacional. Heredia. 95 p.

Dávalos, R., Rodríguez, M. \& Martínez, E. (2008). Almacenamiento de carbono. En: Agroecosistemas cafetaleros de Veracruz. Capítulo 16 (p. 223-233). México: Instituto Nacional de Ecología.

Delgado, L., Acevedo, M., Castellanos, H., Ramírez, H. \& Serrano, J. (2005). Relaciones alométricas y patrones de crecimiento para especies de árboles de la reserva forestal Imataca, Venezuela. INCI. Mayo, Vol. 30, No. 5, p. 275-283. ISSN 03781844.

González, M. \& González, G. (1973). Propiedades fisicas, mecánicas, usos y otras caractrísticas de algunas maderas comercialmente importantes en Costa Rica. Parte I. Laboratorio de Productos Forestales. Universidad de Costa Rica.

González, G., López, A., Rivera, D., Méndez, L., Bonilla, L., Carpio, I. \& Muñoz, A. (198I). Propiedades y usos de 48 especies maderables de llanos del Cortes, Guanacaste. Laboratorio de Productos Forestales. Universidad de Costa Rica.

IPCC. (2007). Cambio climático 2007: Informe de síntesis. Contribución de los Grupos de trabajo I, II y III al Cuarto Informe de evaluación del Grupo Intergubernamental de Expertos sobre el Cambio Climático [Equipo de redacción principal: Pachauri, R.K. y Reisinger, A. (directores de la publicación)]. Ginebra: IPCC, 104 p.

Llach, L. (197I). Propiedades físicas y mecánicas de ciento trece especies. Informe FO-UNDP/SF PAN/6. FAO. Panamá.

Muggeo, V. (2008). Segmented: An R Package to fit regression models with broken-line relationships. The Newsletter of the R Project, Vol. 8/I, p. 20-25.

Neter, J., Kutner, M., Nachtsheim, C. \& Wasserman, W. (1996). Applied linear statistical models. US: McGraw-Hill.

Organización de las Naciones Unidas para la Agricultura y la Alimentación (FAO). (1998). Directrices para la evaluación en los países tropicales y subtropicales. Departamento de Montes. Roma.

Programa de las Naciones Unidas para el Desarrollo (PNUD) y Organización de las Naciones Unidas para la Agricultura y la Alimentación (FAO). (1973). Investigación sobre el formato de la producción de los bosques de noreste: Propiedades y usos de 15 especies maderables del noreste de Nicaragua. Nicaragua. 


\section{Anexo}

Cuadro A I. Distribución del género, especie y densidad de la madera de los árboles en los dos bosques estudiados

\begin{tabular}{|c|c|c|c|}
\hline Género & Especie & Frec. & Densidad $(\delta)$ \\
\hline Anacardium & Excelsum & 9 & 0,38 \\
\hline Andira & Inermis & 4 & 0,27 \\
\hline Apeiba & & 29 & 0,27 \\
\hline Brosimum & & 31 & 0,54 \\
\hline Carapa & Guianensis & 6 & 0,47 \\
\hline Casseria & & 29 & 0,66 \\
\hline Chrysophyllum & Venezuelanense & 4 & 0,47 \\
\hline Coccoloba & & 1 & 0,53 \\
\hline Cordia & & 43 & 0,44 \\
\hline Dendropanax & & 47 & 0,41 \\
\hline Dussia & & 13 & 0,46 \\
\hline Eschweilera & & 7 & 0,75 \\
\hline Eugenia & sp. & 3 & 0,84 \\
\hline Goethalsia & Meiantha & 1 & 0,35 \\
\hline Guazuma & Ulmifolia & । & 0,51 \\
\hline Hasseltia & Floribunda & 7 & 0,54 \\
\hline Hernandia & Didymantha & 3 & 0,25 \\
\hline Hirtella & & 6 & 0,79 \\
\hline Hura & Crepitans & 3 & 0,34 \\
\hline Inga & & 420 & 0,56 \\
\hline Laetia & Procera & 31 & 0,63 \\
\hline Licania & & 5 & 0,64 \\
\hline Luehea & & 24 & 0,47 \\
\hline Minquartia & Guianensis & 2 & 0,61 \\
\hline Naucleopsis & Naga & 2 & 0,38 \\
\hline Ochroma & Pyramidale & 1 & 0,13 \\
\hline Ocotea & & 139 & 0,30 \\
\hline Pentaclethra & Macroloba & 201 & 0,49 \\
\hline Persea & & 22 & 0,53 \\
\hline Poulsenia & Armata & 22 & 0,33 \\
\hline Pouteria & & 27 & 0,67 \\
\hline Pseudolmedia & & 10 & 0,59 \\
\hline Sapium & Allenii & 34 & 0,50 \\
\hline Simarouba & Amara & 1 & 0,32 \\
\hline Spondias & Mombin & 88 & 0,36 \\
\hline Sterculia & & 8 & 0,43 \\
\hline Stryphnodendron & Microstachyum & 2 & 0,34 \\
\hline Symphonia & Globulifera & 14 & 0,56 \\
\hline Terminalia & & 2 & 0,68 \\
\hline Trichilia & & 20 & 0,44 \\
\hline Virola & & 36 & 0,37 \\
\hline Vochysia & Guatemalensis & 2 & 0,35 \\
\hline Xylosma & sp. & 2 & 0,65 \\
\hline
\end{tabular}

Fuente: Laboratorio de Productos Forestales, Universidad de Costa Rica. 
Figura A I. Gráficos para la verificación de supuestos (incluye los dos puntos de cambio, dap= $30 \mathrm{~cm}$ y dap= $50 \mathrm{~cm}$ )

biomasa transformada contra DAP

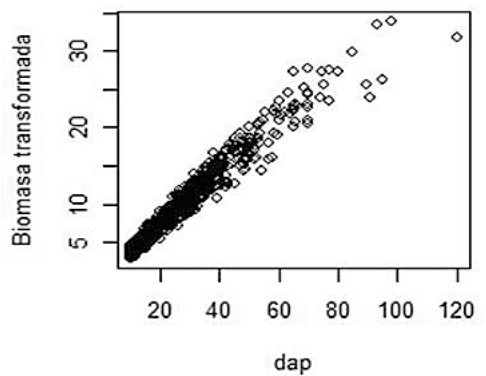

QQ-plot estudentizados

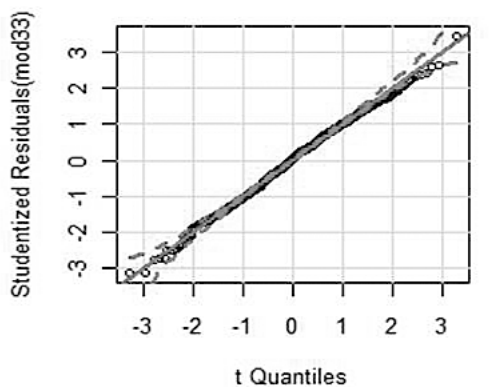

Residuales contra los y ajustados

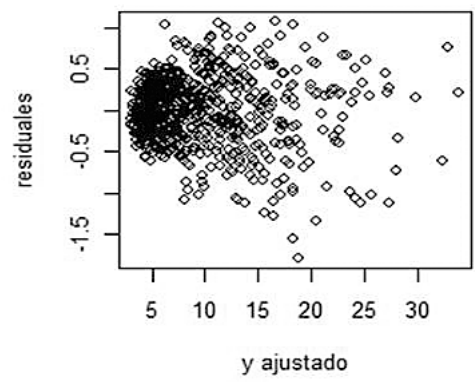

QQ-plot

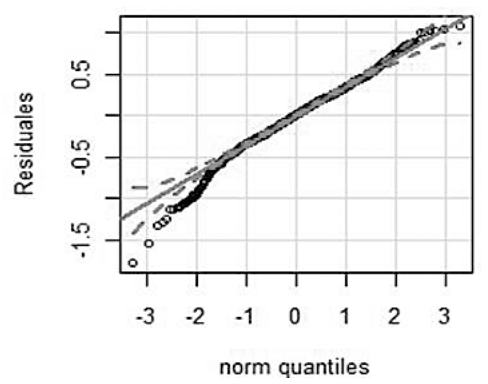

Figura A 2.. Gráficos para la verificación de supuestos (punto de cambio, dap=50 cm)

Residuales contra los y ajustados

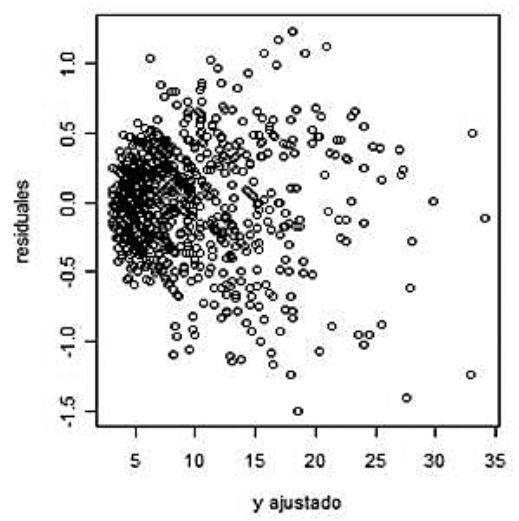

QQ-plot estudentizados

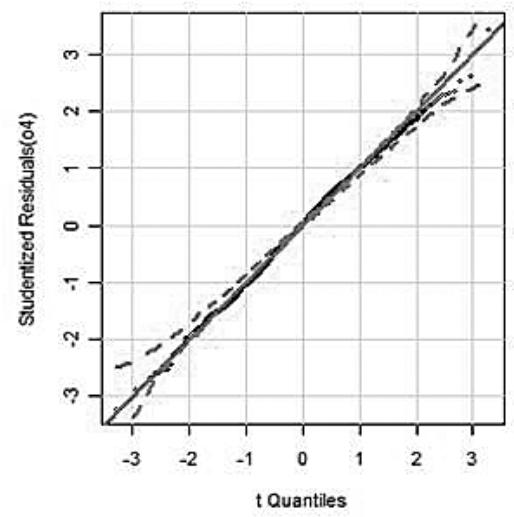

QQ-plot

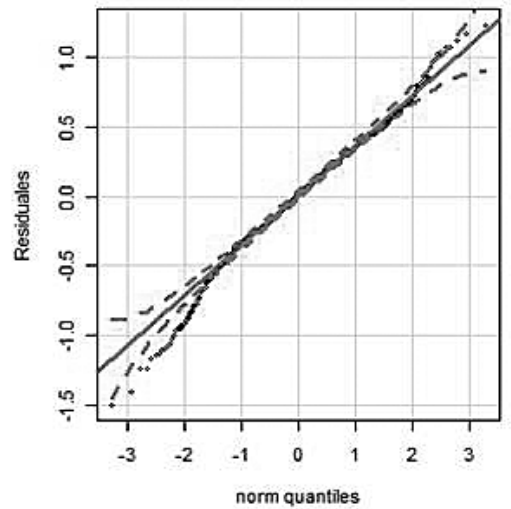

